\title{
Theory of Connexes. II
}

\author{
By
}

\author{
Yōhei YAMASAKI*
}

\section{Introduction}

Here we have a display of the famous game named Hex, where two players White and Black occupy the vertices in the rhombus and who obtains a path between his initially posed pieces wins. It is remarkable that this game always gives a single winner. Regarding the board as the upper half of the sphere, we notice the following statement:

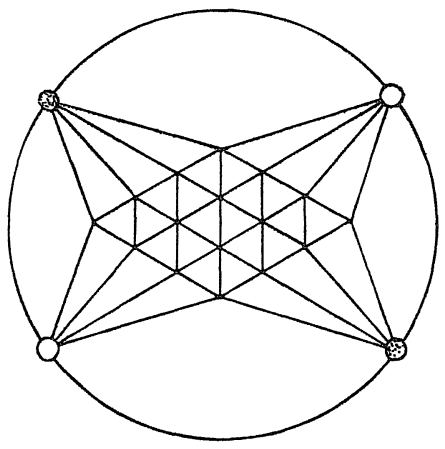

Figure 1

Suppose there be a simplicial decomposition of the sphere invariant by the antipodal mapping. If two players occupy whole the dipoles of vertices, then there exists strictly one player who obtains in his territory a connected set of vertices invariant under the antipodal action.

Our purpose in this paper is to prove the above statement in more general situation. We have already proved in [3] the converse of the relevant statement, namely, a graph with an action of $\boldsymbol{Z}_{2}$ is essentially spherical besides certain exceptions if it admits the unique winner property.

Communicated by S. Hitotumatu, April 17, 1984.

* College of Nursing and Bio-medical Technology, Osaka University, Toyonaka, Osaka 560 , Japan. 


\section{§1. Preliminary}

We fix a set $\Pi$ of two players $T$ and $\perp$ and the involution ${ }^{\wedge}$ of $\Pi$ namely, $\hat{\top}=\perp$ and $\hat{\perp}=\top$. For any finite set $X$, we call a mapping $\mathrm{D}$ from $X$ to $\Pi$ as a division on $X$. We consider a compact real 2-dimensional manifold $M$ with an action of a finite group $G$. We consider also a $G$-invariant simplicial decomposition $\mathbf{K}=\left(K^{0}, K^{1}, K^{2}\right)$ of $M$.

For $i=0$ or 1 , we say two $i$-simplices to be adjacent if they are distinct and are contained in the boundary of an $i+1$-simplex. The connectivity of a subset of $K^{i}$ is considered with respect to this adjacency. We assume that the action of $G$ is faithful on $K^{0}$ and that any complete subset of $K^{0}$ is contained in the boundary of 2-simplex.

For a subset $A$ of $K^{0}$, we denote by [A] the subset of $M$ defined as follows:

$$
[A]=\{x \in M \mid x \text { is a point of a simplex whose vertices are all in } A\} .
$$

Let $X$ be a subset of $M$. Then we denote by $\bar{X}$ the closure of $X$ and define a subgroup $S(X)$ of $G$ as

$$
S(X)=\{g \in G \mid X \text { is } g \text {-invariant }\} .
$$

Lemma 1. Let $A$ be a subset of $K^{0}$. Then $S([A])$ coincides with $S(A)$. Let $B$ be a connected component of $A$. Then $[B]$ is a connected component of [A].

The proof of this lemma is easy and is omitted.

Let $D$ be a $G$-invariant division on $K^{0}$. Then we denote by $\mathscr{B}$ the set of connected components of the open set $M-\cup_{\pi \in \Pi}\left[\mathfrak{D}^{-1}(\pi)\right]$. We fix the division $D$ in the rest of this section. We assume that $D$ is not constant.

Lemma 2. Let $\pi$ be a player, $C$ a connected component of $\mathfrak{D}^{-1}(\pi)$ and $\mathscr{B}_{C}$ a subset of $\mathscr{B}$ defined as follows:

$$
\mathscr{B}_{C}=\left\{\not \subset \in \mathscr{B} \mid \bar{b} \cap \mathfrak{D}^{-1}(\pi) \subset C\right\} .
$$

Assume there be given an element $\measuredangle_{0}$ of $\mathscr{B}_{C}$. Then

$$
S(C)=\left\{g \in G \mid g b_{0} \in \mathscr{B}_{C}\right\} \text {. }
$$

Especially, $S(C)$ contains $S\left(\measuredangle_{0}\right)$.

This lemma follows immediately the above lemma and the facts that $D$ is $G$-invariant and that $C$ is a connected component.

Lemma 3. An element of $\mathscr{B}$ is orientable. 
Proof. Let $b$ be an element of $\mathscr{B}$. Then for any 1 -simplex in $b$, there exist exactly two 1 -simplices adjacent to it. Touring along the 1 -simplices of $b$, we obtain an orientation with the 0 -simplices occupied by $T$ on the right side.

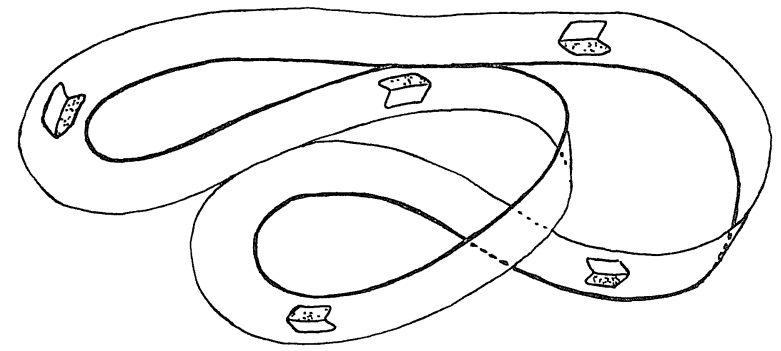

Figure 2

Lemma 4. Let $\not$ be an element of $\mathscr{B}$ and $S_{0}(\not)$ the set consisting of the elements of $S(b)$ which preserve an orientation of $\measuredangle$. Then $S_{0}(\measuredangle)$ is a cyclic subgroup of $S(\measuredangle)$ of index 1 or 2 . If this index is 2 , then any element of $S(\measuredangle)$ $-S_{0}(\measuredangle)$ stabilizes exactly two elements of $K^{1} \cup K^{2}$ contained in $\measuredangle$.

Proof. Let $\Gamma$ be the graph whose vertices are the 1-simplices contained in $\measuredangle$ and the adjacency be defined before. Then there is a natural homomorphism from $S(\mathscr{G})$ to the automorphism group of $\Gamma$, which is injective because $G$ is faithful on $K^{0}$. Now our lemma is clear.

Lemma 5. Let $\measuredangle$ be an element of $\mathscr{B}$. Then for each player $\pi, \mathfrak{D}^{-1}(\pi) \cap \bar{Z}$ is connected and is invariant under $S(6)$.

This lemma is easily verified and its proof is omitted.

Let $\pi$ be a player and $C$ a connected component of $b^{-1}(\pi)$. We define a family $\mathscr{N}_{C}$ of connected components of $\mathfrak{D}^{-1}(\hat{\pi})$ as follows:

$\mathscr{N}_{C}=\left\{E \mid E\right.$ is a connected components of $\mathfrak{D}^{-1}(\hat{\pi})$ such that $C \cup E$ is connected $\}$. For $E \in \mathscr{N}_{C}$ we define a subset of $G$ as follows:

$$
\left(\begin{array}{c}
C \\
E
\end{array}\right)=\left\{g \in G \mid g E \in \mathscr{N}_{C}\right\} .
$$

We define also a new division $\mathfrak{D}_{C}$ on $K^{0}$ as follows:

$$
\mathfrak{D}_{C}^{-1}(\pi)=\mathfrak{D}^{-1}(\pi)-G C \text {. }
$$

Lemma 6. Let the assumptions be as above. Assume moreover that D is not constant. Then the stabilizer of the connected component $C^{\prime}$ of $D_{C}{ }^{-1}(\hat{\pi})$ containing $C$ is given as follows: 


$$
S\left(C^{\prime}\right)=\left\langle\left(\begin{array}{c}
C \\
E
\end{array}\right) \mid E \in \mathscr{N}_{C}\right\rangle
$$

If $\left|\mathscr{N}_{C}\right|=1$, moreover, then $S\left(C^{\prime}\right)=S(E)$, where $E$ is the element of $\mathscr{N}_{C}$.

Proof. It is evident that $S\left(C^{\prime}\right)$ contains the relevant group. Let $\gamma$ be an element of $S\left(C^{\prime}\right)$. Then there exists a series $\gamma_{1} C, g_{1} E_{1}, \gamma_{2} C, g_{2} E_{2}, \ldots, \gamma_{n-1} C$, $g_{n-1} E_{n-1}, \gamma_{n} C$ for a positive integer $n$ where $E_{i} \in \mathscr{N}_{C}, g_{i} \in G, \gamma_{i} \in G, \gamma_{1}{ }^{-1} \gamma_{n}=\gamma$ and the union of each neighbouring two is connected. If $n=1$, then $\gamma_{1}^{-1} \gamma_{n} \in S(C)$. If $n \geqq 2$, then for $1 \leqq i \leqq n-1$

$$
\gamma_{i}^{-1} g_{i} \text { and } \gamma_{i+1}^{-1} g_{i} \in\left(\begin{array}{c}
C \\
E_{i}
\end{array}\right) .
$$

Therefore $\gamma=\gamma_{1}{ }^{-1} \gamma_{n}$ is an element of the relevant group. The latter part is evident.

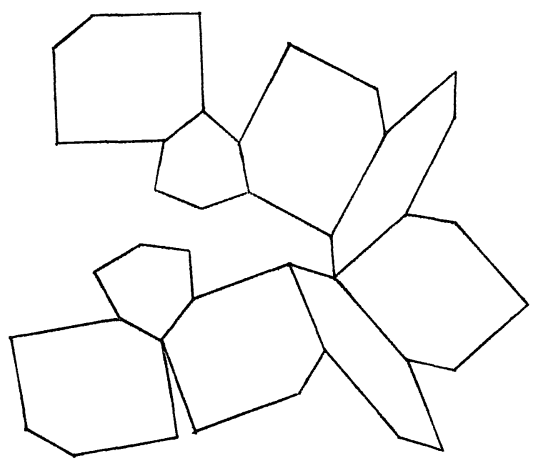

Figure 3

\section{§2. Linear Groups on the Unit Sphere (1)}

From now on we assume $M$ as the unit sphere in $\boldsymbol{R}^{3}$. For a positive integer $n$ we define $3 \times 3$-matrices $g_{-}(n)$ and $g_{+}$as follows:

$$
\begin{gathered}
g_{-}(n)=\left(\begin{array}{rcc}
\cos \frac{\pi}{n} & \sin \frac{\pi}{n} & 0 \\
-\sin \frac{\pi}{n} & \cos \frac{\pi}{n} & 0 \\
0 & 0 & -1
\end{array}\right) \\
g_{+}=\left(\begin{array}{rrr}
1 & 0 & 0 \\
0 & -1 & 0 \\
0 & 0 & -1
\end{array}\right) .
\end{gathered}
$$


In this section we assume $G$ as one of the linear groups $G_{-}(n)=\left\langle g_{-}(n)\right\rangle$ and $G_{+}(n)=\left\langle g_{-}(n)^{2}, g_{+}\right\rangle$with the usual action on $M$. In case $G=G_{+}(n)$, we assume $n \geqq 2$. We fix a $G$-invariant simplicial decomposition $\mathbb{K}=\left(K^{0}, K^{1}, K^{2}\right)$ of $M$.

Theorem. Let the assumptions be as above. Let $\mathrm{D}$ be a G-invariant division on $K^{0}$. We regard a player $\pi$ as a winner if $\mathrm{D}^{-1}(\pi)$ has a G-invariant connected component. Then there exists a unique winner.

Proof. This statement is obvious if $\mathfrak{D}$ is a constant mapping. Suppose it to be false and let $\mathrm{D}$ be a counter example minimal with respect to the number $|\mathscr{B}|$. We choose a pair $(\measuredangle,[C])$ of an element $\measuredangle$ of $\mathscr{B}$ and a connected component $[C]$ of $M-b$ such that $[C]$ is minimal. Then, by the Jordan curve theorem, $b$ is the only element of $\mathscr{B}$ whose closure intersects with $C$. Lemma 2 tells us $S(C)=S(\measuredangle)$. If $G=G_{+}(n)$ for $n \geqq 2$, then

$$
S(C)=S(\measuredangle)=S_{0}(\measuredangle),
$$

and if $G=G_{-}(n)$, then by Lemma 4

$$
S(C)=S(\measuredangle) \not \supset g_{-}(n) \text {. }
$$

In any way, we have $S(C) \neq G$.

Now we consider a division $\mathfrak{b}_{C}$ with respect to the player $\pi=\mathfrak{D}(C)$. Then $\mathfrak{D}_{C}{ }^{-1}(\pi) \subset \mathfrak{D}^{-1}(\pi)$. We have seen above that there is no $G$-invariant connected component of $D^{-1}(\pi)$ besides the ones of $D_{C}{ }^{-1}(\pi)$. On the other hand, by Lemma 6 , every $G$-invariant connected component $D_{C}{ }^{-1}(\hat{\pi})$ remains a connected component even if it is restricted to $D^{-1}(\hat{\pi})$. This contradicts the minimality of $D$.

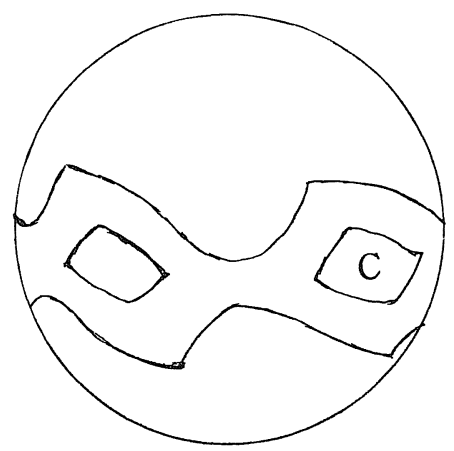

Figure 4 


\section{§3. Linear Groups on the Unit Sphere (2)}

In the last section we have studied the action of $G_{-}(n)$ and $G_{+}(n)$ on the unit sphere. We know that the finite linear group of degree 3 is conjugate in $\mathrm{SL}(3, \mathbf{R})$ to a subgroup of $\left\langle g_{-}(n), g_{+}\right\rangle$for a positive integer $n$ or of polyhedral groups. Then it is still possible that the simplicial decomposition in the last section admits an action of larger groups. We give here an example.

Let $G$ be the group generated by the reflections on $x y$-, $y z$ - and $z x$-planes, which contains $G_{-}(1)$. Let $\mathbf{K}=\left(K^{0}, K^{1}, K^{2}\right)$ be a $G$-invariant simplicial decomposition of $M$. Let $\mathfrak{D}$ be a $G$-invariant division on $K^{0}$. Then one of $\mathfrak{D}^{-1}(T)$ and $\mathfrak{D}^{-1}(\perp)$ has a $G_{-}(1)$ invariant connected component by our theorem, which is $G$-invariant. This causes the following proposition.

Proposition. Let $\mathbf{K}=\left(K^{0}, K^{1}, K^{2}\right)$ be a simplicial decomposition of a triangle and $\mathfrak{D}$ a division on $K^{0}$. Then exactly one of $\mathfrak{D}^{-1}(T)$ and $\mathfrak{D}^{-1}(\perp)$ contains a connected components which intersects each edge of the initial triangle.

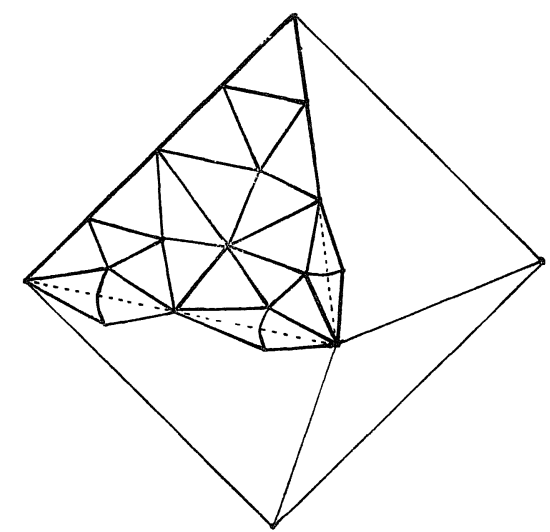

Figure 5

If the initial triangle is on a plane and any 1-simplex is parallel to an edge of the previons triangle, then this example is equivalent to what Komiya [1] calls trinitrix, which was announced to the author by his friend Mr. Tsujino. 


\section{Bibliography}

[1] Komiya, K., A connection game on a triangle and a numbering puzzle, Surikagaku Puzzle V, 1980, in Japanese.

12] Yamasaki, Y., Theory of division games, Publ. RIMS Kyoto Univ., 14 (1978), 337358.

[3] - Theory of connexes. I, Publ. RIMS Kyoto Univ., 17 (1981), 777-812. 
